\title{
NON-CLASSICAL GODEAUX SURFACES
}

\author{
CHRISTIAN LIEDTKE
}

\begin{abstract}
A non-classical Godeaux surface is a minimal surface of general type with $\chi=K^{2}=1$ but with $h^{01} \neq 0$. We prove that such surfaces fulfill $h^{01}=1$ and they can exist only over fields of positive characteristic at most 5. Like non-classical Enriques surfaces they fall into two classes: the singular and the supersingular ones. We give a complete classification in characteristic 5 and compute their Hodge-, Hodge-Witt- and crystalline cohomology (including torsion). Finally, we give an example of a supersingular Godeaux surface in characteristic 5 .
\end{abstract}

\section{CONTENTS}

Introduction

1. Numerical Godeaux surfaces

2. Non-classical Godeaux surfaces

3. Supersingularity and global 1-forms

4. Picard scheme and Hodge invariants

5. Classification and examples

References

\section{INTRODUCTION}

Among minimal surfaces of general type, the ones with the smallest invariants possible are those with $K^{2}=1$ and $h^{01}=p_{g}=0$. The first example of such a surface has been constructed by Godeaux [Go] in 1931. Since then, there is an extensive search for such surfaces and up to today there is still no complete classification of them. We refer to [BHPV], Chapter VII.10] for details and further references.

Definition. A minimal surface of general type with $\chi\left(\mathcal{O}_{X}\right)=K_{X}^{2}=1$ is called a numerical Godeaux surface. A numerical Godeaux surface with $h^{01}(X) \neq 0$ is called non-classical.

Proposition. The first Betti number of a numerical Godeaux surface is zero. Non-classical Godeaux surfaces fulfill $h^{01}=p_{g}=1$.

This means that the Picard scheme of a non-classical Godeaux surface is not reduced, which can happen over fields of positive characteristic only. In particular, a numerical Godeaux surface in characteristic zero fulfills $h^{01}=p_{g}=0$. On the other hand, Miranda [Mir] has given an example of a non-classical Godeaux surface in characteristic 5. Our first main result is the following

Theorem. Non-classical Godeaux surfaces can exist in characteristic $2 \leq p \leq 5$ only.

Date: April 21, 2008. revised: August 4, 2008.

2000 Mathematics Subject Classification. 14J29, 14J10. 
This should be compared with Enriques surfaces, i.e., minimal surfaces of Kodaira dimension zero with $\chi\left(\mathcal{O}_{X}\right)=1$. The classical ones have $h^{01}=p_{g}=0$, whereas the non-classical ones fulfill $h^{01}=p_{g}=1$. Non-classical Enriques surfaces exist in characteristic 2 only [BM2, Theorem 5].

Like Enriques surfaces, all global 1-forms on a numerical Godeaux surface are $d$-closed. Another feature of Enriques surfaces is that their second crystalline or étale cohomology is spanned by algebraic cycles, i.e., that they are supersingular in the sense of Shioda. This is also true for numerical Godeaux surfaces if the surface lifts to characteristic zero or if a conjecture of Artin and Mazur holds true, cf. Section 3 .

Since $H^{1}\left(\mathcal{O}_{X}\right)$ of a non-classical Godeaux surface is one-dimensional, the action of Frobenius on it is either zero or bijective. In analogy with Enriques surfaces [BM3, Section 3] we define

Definition. A numerical Godeaux surface is called

$$
\begin{array}{ll}
\text { classical } & \text { if } p_{g}=h^{01}=0, \\
\text { singular } & \text { if } p_{g}=h^{01}=1 \text { and } F \text { is bijective on } H^{1}\left(\mathcal{O}_{X}\right), \text { and } \\
\text { supersingular } & \text { if } p_{g}=h^{01}=1 \text { and } F \text { is zero on } H^{1}\left(\mathcal{O}_{X}\right) .
\end{array}
$$

Now we specialise to characteristic 5 , the largest characteristic possible for non-classical Godeaux surfaces. As already mentioned, the main feature is the non-reducedness of their Picard schemes. More precisely, we show

Theorem. Let $X$ be a non-classical Godeaux surface in characteristic 5 . Then

$$
\begin{aligned}
& X \text { singular } \quad \text { implies } \operatorname{Pic}^{\tau}(X)=\operatorname{Pic}^{0}(X) \cong \mu_{5} \text {, and } \\
& X \text { supersingular implies } \operatorname{Pic}^{\tau}(X)=\operatorname{Pic}^{0}(X) \cong \alpha_{5} .
\end{aligned}
$$

This result is the key to determining the Hodge-, the Hodge-Witt- and the crystalline cohomology groups (including torsion) of non-classical Godeaux surfaces in characteristic 5. Also the degeneration behaviour of the Frölicher- and the slope spectral sequence are answered. A picture emerges that is very similar to non-classical Enriques surfaces. We refer to Section 4 for precise statements.

Next, we prove that every non-classical Godeaux surface in characteristic 5 arises as the quotient of a quintic surface in $\mathbb{P}^{3}$ by a linear and fixed-point free action of $\mathbb{Z} / 5 \mathbb{Z}$, resp. $\alpha_{5}$, i.e., every such surface is obtained by a characteristic 5-version of Godeaux's original construction. This result is analogous to Reid's classification [Rei, Section 1] of numerical Godeaux surfaces with 5-torsion over the complex numbers.

Theorem. Let $X_{\mathrm{can}}$ be the canonical model of a non-classical Godeaux surface in characteristic 5. Then its associated $\mathbb{Z} / 5 \mathbb{Z}$-torsor (in the singular case), resp. $\alpha_{5}$-torsor (in the supersingular case) is isomorphic to a quintic surface in $\mathbb{P}^{3}$. Moreover, the $\mathbb{Z} / 5 \mathbb{Z}$-, resp. $\alpha_{5}$-action on this quintic extends to a linear action on the ambient $\mathbb{P}^{3}$.

Finally, it remains to establish existence of these surfaces. Miranda's surface [Mir] mentioned above is a singular Godeaux surface in characteristic 5 and we end this article by proving

Theorem. There do exist supersingular Godeaux surfaces in characteristic 5.

Acknowledgements. I thank Stefan Schröer for interesting discussions and help. Also, I thank the referee for pointing out a couple of inaccuracies and a mistake in the first version of this article.

\section{Numerical GodeauX SURFACES}

A minimal surface $X$ of general type defined over an arbitrary algebraically closed field fulfills $K_{X}^{2} \geq 1$. We first recall a couple of general facts about surfaces for which the equality $K_{X}^{2}=1$ 
holds. In particular, we will see that $\chi\left(\mathcal{O}_{X}\right) \geq 1$ holds true. Hence surfaces with $K_{X}^{2}=\chi\left(\mathcal{O}_{X}\right)=1$, the so-called numerical Godeaux surfaces, have the lowest invariants possible among all minimal surfaces of general type. Although the first Betti number of such a surface is always zero, over fields of positive characteristic we only have $h^{01} \leq 1$, which leads to the notion of non-classical Godeaux surfaces.

We start with some general facts that are well known over the complex numbers, cf. Sections 10 and 11 of $[$ Bo]. However, a little care is needed in positive characteristic.

Proposition 1.1. Let $X$ be a minimal surface of general type with $K_{X}^{2}=1$. Then the following equalities and inequalities hold true:

$$
b_{1}(X)=0, \quad\left|\pi_{1}^{\text {ét }}(X)\right| \leq 6, \quad 1 \leq \chi\left(\mathcal{O}_{X}\right) \leq 3, \quad p_{g}(X) \leq 2, \quad \text { and }, \quad h^{01}(X) \leq 1 .
$$

In particular, if $h^{01}(X) \neq 0$ then $X$ has a non-reduced Picard scheme, which can happen in positive characteristic only.

Proof. From Noether's inequality $K^{2} \geq 2 p_{g}-4$, which also holds in positive characteristic by [Lie, Theorem 2.1], we obtain $p_{g} \leq 2$ and then $\chi \leq 3$. Then, $K^{2}+\chi \geq 2$ yields $\chi \geq 1$, cf. [Ek, Corollary II.1.8]. Using the inequalities of [Ek, Corollary II.1.8] again, we see that $h^{01} \leq 1$ if $p_{g}=0$ or $p_{g}=2$. In case $p_{g}=1$, we also must have $h^{01} \leq 1$ since $\chi \geq 1$.

Let $\tilde{X}$ be an étale cover of $X$ of degree $n$. Then $\chi\left(\mathcal{O}_{\tilde{X}}\right)=n \chi\left(\mathcal{O}_{X}\right) \geq n$, which implies $p_{g}(\tilde{X}) \geq$ $n-1$. We also have $K_{\tilde{X}}^{2}=n$ and then Noether's inequality $K^{2} \geq 2 p_{g}-4$ implies $n \leq 6$. Hence the étale fundamental group of $X$ has order at most 6 , which implies that the first Betti number of $X$ is zero.

Since we are interested in surfaces of general type with the lowest invariants possible, the previous result tells us to look at the following class of surfaces:

Definition 1.2. A numerical Godeaux surface is a minimal surface of general type with $K_{X}^{2}=1$ and $\chi\left(\mathcal{O}_{X}\right)=1$.

If a numerical Godeaux surface fulfills $p_{g}=h^{01} \neq 0$ we are in positive characteristic and it makes sense to look at the action $F$ of Frobenius on $H^{1}\left(\mathcal{O}_{X}\right)$, which is either zero or bijective since this space is one-dimensional. In analogy with Enriques surfaces [BM3, Section 3] we define:

Definition 1.3. A numerical Godeaux surface is called

$$
\begin{array}{ll}
\text { classical } & \text { if } p_{g}=h^{01}=0, \\
\text { singular } & \text { if } p_{g}=h^{01}=1 \text { and } F \text { is bijective on } H^{1}\left(\mathcal{O}_{X}\right), \text { and } \\
\text { supersingular } & \text { if } p_{g}=h^{01}=1 \text { and } F \text { is zero on } H^{1}\left(\mathcal{O}_{X}\right) .
\end{array}
$$

We will refer to the last two classes as non-classical Godeaux surfaces.

By Proposition 1.1, every numerical Godeaux surface in characteristic zero is classical.

Godeaux's original construction [Go] of a classical Godeaux surface is the quotient of a smooth quintic in $\mathbb{P}^{3}$ by a fixed point free action of $\mu_{5}$, which works over arbitrary fields of characteristic $p \neq 5$. Lang $[\overline{\mathrm{La}}]$ also constructed classical Godeaux surfaces in characteristic 5.

Theorem 1.4 (Godeaux, Lang). There do exist classical Godeaux surfaces in every characteristic.

We remark that the classification of numerical Godeaux surfaces even over the complex numbers is still incomplete, and refer to [BHPV], Chapter VII.10] for details and further references. 


\section{NON-CLASSICAL GODEAUX SURFACES}

In the previous section we have seen that classical numerical Godeaux surfaces exist in every characteristic. In this section we show that non-classical Godeaux surfaces can only exist in characteristic at most 5. This is rather easy for the singular Godeaux surfaces but much more complicated for the supersingular Godeaux surfaces.

Theorem 2.1. Singular Godeaux surfaces can exist in characteristic $2 \leq p \leq 5$ only. These surfaces are not weakly unirational.

Proof. Let $X$ be a singular Godeaux surface. Since $H^{1}\left(\mathcal{O}_{X}\right)$ is one-dimensional and Frobenius acts injectively, we obtain an inclusion of the group scheme $\mu_{p}$ into $\operatorname{Pic}(X)$. This defines a $\mu_{p}^{D}$-torsor over $X$, where $-{ }^{D}:=\operatorname{Hom}\left(-, \mathbb{G}_{m}\right)$ denotes Cartier duality. Since $\mu_{p}^{D} \cong \mathbb{Z} / p \mathbb{Z}$ this torsor is just an étale cover of degree $p$ of $X$. Hence $p \leq 6$ by Proposition 1.1

In particular, the algebraic fundamental group has non-trivial $p$-torsion. By [ $\overline{\mathrm{Cr}}$, Theorem 2.5], such a surface cannot be weakly unirational.

Remark 2.2. Shioda has given examples of classical Godeaux surfaces that are weakly unirational in every positive characteristic $p \neq 5$ with $p \not \equiv 1 \bmod 5$, cf. [Sh2, Proposition 5].

What makes the analysis of supersingular Godeaux surfaces more difficult is the fact that the trivial Frobenius action on $H^{1}\left(\mathcal{O}_{X}\right)$ gives rise to an $\alpha_{p}$-torsor over $X$, whose total space need not be a normal surface. In particular, we cannot apply geometric arguments to this torsor so easily. The main ingredient in the proof of Theorem 2.1 is Noether's inequality which we now establish in a singular context:

Let $X$ be a smooth surface over an algebraically closed field of characteristic $p>0$ and $\pi: Y \rightarrow X$ be a non-trivial $\alpha_{p^{-}}$or $\mu_{p}$-torsor. From the discussion [Ek, Section I] it follows that $Y$ is a possibly non-normal but integral Gorenstein surface. Since the dualising sheaf $\omega_{Y}$ of $Y$ is invertible, we define the self-intersection of the canonical divisor of $Y$ to be

$$
K_{Y}^{2}:=\chi\left(\mathcal{O}_{Y}\right)-2 \chi\left(\omega_{Y}^{-1}\right)-\chi\left(\omega_{Y}^{-2}\right),
$$

which coincides with the usual self-intersection in the smooth case.

In [Ek, Proposition I.1.7] the algebra $\pi_{*} \mathcal{O}_{Y}$ is shown to be a successive extension of invertible sheaves. If $\pi$ is an $\alpha_{p}$-torsor, then these invertible sheaves are isomorphic to $\mathcal{O}_{X}$. Also, $\omega_{Y} \cong \pi^{*}\left(\omega_{X}\right)$ in this case. If $\pi$ is a $\mu_{p}$-torsor, then $\pi_{*} \mathcal{O}_{Y}$ is a successive extension of $\mathcal{L}^{\otimes i}, i=0, \ldots, p-1$ for a suitable invertible sheaf $\mathcal{L}$ with $\mathcal{L}^{\otimes p} \cong \mathcal{O}_{X}$. In this case we have $\omega_{Y} \cong \pi^{*}\left(\omega_{X} \otimes \mathcal{L}^{\otimes(p-1)}\right)$. It follows that the equalities

$$
\chi\left(\mathcal{O}_{Y}\right)=p \chi\left(\mathcal{O}_{X}\right) \quad \text { and } \quad K_{Y}^{2}=p K_{X}^{2}
$$

hold true.

What makes Noether's inequality for $Y$ a little bit tricky is the fact that $Y$ is only an integral Gorenstein scheme, so that linear systems and intersection numbers have to be treated with care.

Proposition 2.3. Let $X$ be a minimal surface of general type and $\pi: Y \rightarrow X$ be a non-trivial $\alpha_{p^{-}}$or $\mu_{p}$-torsor. Then Noether's inequality

$$
K_{Y}^{2} \geq 2 h^{0}\left(\omega_{Y}\right)-4
$$

holds true. If the image of the canonical map is a curve, even $K_{Y}^{2} \geq 2 h^{0}\left(\omega_{Y}\right)-2$ holds true. 
Proof. Since $\pi: Y \rightarrow X$ is purely inseparable of degree $p$, the Frobenius morphism $F$ factors as $\rho: X^{(p)} \rightarrow Y$ followed by $\pi$. We already noted that $\omega_{Y} \cong \pi^{*}\left(\omega_{X} \otimes \mathcal{L}^{\otimes(p-1)}\right)$ for a suitable invertible sheaf $\mathcal{L}$. Using the fact that $\mathcal{L}^{\otimes p} \cong \mathcal{O}_{X}$, we get

$$
\rho^{*} \omega_{Y} \cong F^{*}\left(\omega_{X} \otimes \mathcal{L}^{\otimes(p-1)}\right) \cong \omega_{X^{(p)}}^{\otimes p}
$$

We denote by $L$ the linear system $\rho^{*} H^{0}\left(\omega_{Y}\right)$ of $H^{0}\left(\omega_{X^{(p)}}^{\otimes p}\right)$ and consider the composed (rational) morphism

$$
\varphi:=\varphi_{|L|} \quad: \quad X^{(p)} \stackrel{\rho}{\rightarrow} \quad Y \stackrel{\phi_{1}}{\rightarrow} \quad Z \quad \subseteq \quad \mathbb{P}^{h^{0}\left(\omega_{Y}\right)-1}
$$

where $Z$ denotes the closure of the image of the (rational) canonical map $\phi_{1}$ of $Y$. Being a minimal surface of general type we have $K_{X}^{2} \geq 1$, hence $K_{Y}^{2} \geq p$ and thus we may assume $h^{0}\left(\omega_{Y}\right) \geq 3$, i.e., that $Z$ is a curve or a surface.

Let us first assume that $Z$ is a surface, in which case $\operatorname{deg} \varphi \geq p$. If $\phi_{1}$ is not birational (or $Z$ is not ruled) then [Ek, Proposition 0.1.3], which equally well works for linear subsystems of complete linear systems, yields $h^{0}\left(\omega_{Y}\right)=\operatorname{dim} L \leq\left(p K_{X^{(p)}}\right)^{2} /(2 p)+2=K_{Y}^{2} / 2+2$ and we are done. We may now assume that $\phi_{1}$ is birational (and $Z$ is ruled). For every section of $\omega_{Y}$ we obtain a short exact sequence

$$
\left.0 \rightarrow \mathcal{O}_{Y} \rightarrow \omega_{Y} \rightarrow \omega_{Y}\right|_{D} \rightarrow 0
$$

where $D$ is a Cartier divisor on a Gorenstein scheme. In particular, $D$ is Gorenstein and the adjunction formula holds. The divisor $\rho^{*} D$ lies in $\omega_{X^{(p)}}^{\otimes p}$ and since $h^{1}\left(\omega_{X^{(p)}}^{\otimes(-p)}\right)=0$ by [Ek, Theorem II.1.7], we obtain $h^{0}\left(\mathcal{O}_{\rho^{*} D}\right)=1$. Thus, $h^{0}\left(\mathcal{O}_{D}\right)=1$ and we compute $h^{0}\left(\omega_{D}\right)=1-\chi\left(\mathcal{O}_{D}\right)=1+K_{Y}^{2}$. A generic hyperplane section of $Z$ is an integral curve, and thus corresponds to a Cartier divisor $D$ on $Y$ which has a component $D^{\prime}$, possibly only a Weil divisor, which is an integral curve (as $\phi_{1}$ is an isomorphism over an open and dense subset). We denote by $V$ the image of $H^{0}\left(\omega_{Y}\right) \rightarrow$ $H^{0}\left(D,\left.\omega_{Y}\right|_{D}\right)$. Every non-zero element of $V$ corresponds to a hyperplane section of $Z$ and is thus nontrivial on $D^{\prime}$. Since $D^{\prime}$ is integral, the multiplication of two non-zero sections of an invertible sheaf is non-zero, we can apply the Clifford argument and obtain the estimate $2 h^{0}\left(\omega_{Y}\right)-4=2 \operatorname{dim} V-2 \leq$ $h^{0}\left(D,\left.\omega_{Y}\right|_{D} ^{\otimes 2}\right)-1=h^{0}\left(D, \omega_{D}\right)-1=K_{Y}^{2}$.

We may now assume that $Z$ is a curve. To begin with, we follow the proof of [BHPV] Theorem VII.3.1]. We consider the linear subsystem $L:=\rho^{*} H^{0}\left(\omega_{Y}\right)$ of $H^{0}\left(\omega_{X(p)}^{\otimes p}\right)$ and write this pencil as $|L|=|n F|+V$, where $V$ denotes its fixed part. Then $h^{0}\left(\omega_{Y}\right)=\operatorname{dim} L \leq 1+n$. We compute $K_{Y}^{2}=p K_{X}^{2}=(n F+V) K_{X} \geq n F K_{X}$ and we are done if $F K_{X} \geq 2$. In the remaining cases we either have $F K_{X}=0$ or $F K_{X}=1$. If $F K_{X}=0$ then $F$ is a sum of fundamental cycles and these cannot form a pencil, i.e., this case does not occur. It remains to exclude the possibility $F K_{X}=1$. Since $F$ moves in a pencil we have $F^{2} \geq 0$ and also $F^{2}$ must be an odd integer by the adjunction formula, hence $F^{2} \geq 1$. The Hodge index theorem yields $F^{2} K_{X}^{2} \leq\left(F K_{X}\right)^{2}=1$, which leads to $K_{X}^{2}=F K_{X}=F^{2}=1$.

It remains to deal with this case: since $F^{2}=1$, we have to blow up once $\tilde{X} \rightarrow X$ to obtain a morphism $\tilde{X} \rightarrow \mathbb{P}^{1}$, which is in fact a fibration. The $(-1)$-curve $E \subset \tilde{X}$ dominates $\mathbb{P}^{1}$, i.e., defines a section. The induced $\alpha_{p}$-torsor (resp. $\mu_{p}$-torsor) $\tilde{Y} \rightarrow \tilde{X}$ restricts to a trivial torsor over $E$ because there are no non-trivial such torsors over a rational curve. In particular, we can extend the section of $\tilde{X} \rightarrow \mathbb{P}^{1}$ to $\tilde{Y}$. This means that $\tilde{Y} \rightarrow \mathbb{P}^{1}$ is equal to its Stein factorisation, hence that its fibres are geometrically integral. Hence for every irreducible fibre $F$ of $\tilde{X} \rightarrow \mathbb{P}^{1}$ its inverse image $\pi^{*} F$ is an integral curve. 
We now compute on $X$ : let $F^{\prime}$ be a general member of $|F|$ and set $\tilde{F}^{\prime}:=\pi^{*} F^{\prime}$, which is an integral curve by what we have just proved. We consider the short exact sequence

$$
\left.0 \rightarrow \omega_{Y}\left(-\tilde{F}^{\prime}\right) \rightarrow \omega_{Y} \rightarrow \omega_{Y}\right|_{\tilde{F}^{\prime}} \rightarrow 0
$$

Taking cohomology and using Serre duality, we get $h^{1}\left(\left.\omega_{Y}\right|_{\tilde{F}^{\prime}}\right) \neq 0$. Since $\tilde{F}^{\prime}$ is an integral curve, Clifford's inequality gives $h^{0}\left(\left.\omega_{Y}\right|_{\tilde{F}^{\prime}}\right) \leq 1+\left(\left.\operatorname{deg} \omega_{Y}\right|_{\tilde{F}^{\prime}}\right) / 2=1+K_{Y}^{2} / 2$. Now, $\mathcal{B}:=\omega_{Y}\left(-\tilde{F}^{\prime}\right)$ is the pull-back of a numerically trivial invertible sheaf on $X$, and hence $h^{0}(\mathcal{B}) \leq 1$. From this we get Noether's inequality.

In case $h^{0}(\mathcal{B})=1$, the curve $\tilde{F}^{\prime}$ is a reduced section of $\omega_{Y}$ and so the map associated to $\omega_{Y}$ is not composed with a pencil. Hence if the image of the canonical map is a curve, we have $h^{0}(\mathcal{B})=0$ and the stronger inequality $h^{0}\left(\omega_{Y}\right) \leq 1+K_{Y}^{2} / 2$ holds true.

Theorem 2.4. Supersingular Godeaux surfaces can exist in characteristic $2 \leq p \leq 5$ only.

ProOF. Let $X$ be a supersingular Godeaux surface. By definition, the Frobenius-action on the one-dimensional vector space $H^{1}\left(\mathcal{O}_{X}\right)$ is trivial. This action gives rise to a non-trivial $\alpha_{p}$-torsor $\pi: Y \rightarrow X$. By (2) we have $K_{Y}^{2}=p K_{X}^{2}=p$ and $\chi\left(\mathcal{O}_{Y}\right)=p \chi\left(\mathcal{O}_{X}\right)=p$. The latter equality gives $h^{0}\left(\omega_{Y}\right) \geq p-1$. By Proposition 2.3 , the inequality $p \geq 2(p-1)-4$ has to be fulfilled and we obtain $p \leq 5$.

Remark 2.5. The same argument as in [La, Corollary 1 of Theorem 1] shows that the total space of the $\alpha_{p}$-torsor associated to a supersingular Godeaux surface is never a smooth surface.

Corollary 2.6. Non-classical Godeaux surfaces can exist in characteristic $2 \leq p \leq 5$ only.

\section{SUPERSINGULARITY AND GLOBAL 1-FORMS}

In this section we show that numerical Godeaux surfaces that are liftable to characteristic zero are supersingular in the sense of Shioda. Without the lifting assumption we can show this result only modulo a conjecture of Artin and Mazur. We finish by showing that global 1-forms on a numerical Godeaux surface are always $d$-closed.

By a theorem of Igusa, the rank of the Néron-Severi group of a smooth projective surface is less or equal to its second Betti number. This led Shioda $[\overline{\mathrm{Sh}}]$ to introduce the following notion:

Definition 3.1. A smooth and projective surface is called supersingular in the sense of Shioda if the rank of its Néron-Severi group is equal to its second Betti number.

Over fields of characteristic zero, a smooth projective surface is supersingular in the sense of Shioda if and only if $p_{g}=0$ by [Sh2, Section 2].

Proposition 3.2. If a numerical Godeaux surfaces is defined over a field of characteristic zero or if it lifts to characteristic zero then it is supersingular in the sense of Shioda.

PROOF. In characteristic zero we have $p_{g}=0$ and the result follows from [Sh2, Section 2].

If a lifting of $X$ to characteristic zero exists, then the lifted surface $X^{*}$ also has $K^{2}=\chi=1$. Hence $p_{g}\left(X^{*}\right)=0$ by Proposition 1.1 and thus $X^{*}$ is supersingular in the sense of Shioda. We denote by $\rho$ the rank of the Néron-Severi group and conclude as in [Sh2, Lemma 1]

$$
b_{2}\left(X^{*}\right)=b_{2}(X) \geq \rho(X) \geq \rho\left(X^{*}\right)=b_{2}\left(X^{*}\right) .
$$

Hence $\rho(X)=b_{2}(X)$, i.e., $X$ is supersingular in the sense of Shioda. 
If a surface over an algebraically closed field of positive characteristic is supersingular in the sense of Shioda, then the Frobenius-action on its second crystalline cohomology modulo torsion is of slope one, cf. [II, Proposition II.5.12].

Artin and Mazur conjectured that the converse is also true, cf. [II, Remarque II.5.13]. For example, this is true for Enriques surfaces [BM3, Theorem 4]. Modulo this conjecture, numerical Godeaux surfaces are supersingular in the sense of Shioda:

Proposition 3.3. In positive characteristic, the second crystalline cohomology modulo torsion of a numerical Godeaux surface is of slope one.

Proof. As in the proof of [II], Proposition II.7.3.2] one first shows that the Witt vector cohomology $H^{2}\left(W \mathcal{O}_{X}\right)$ is equal to its $V$-torsion. Now, this $V$-torsion is a $W$-module of finite length since it can be related to the Dieudonné-module of some finite flat group scheme, cf. [II, Remarque II.6.4]. Hence $H^{2}\left(W \mathcal{O}_{X}\right)$ is a $W$-module of finite length, which implies that $H_{\text {cris }}^{2}(X / W)$ modulo torsion is of slope one, cf. [II], Formula II.(3.5.4)].

As in [II, Corollaire II.7.3.3] we obtain

Proposition 3.4. The slope spectral sequence of a numerical Godeaux surface degenerates at $E_{1}$ level. In particular, every global 1-form on a numerical Godeaux surface is d-closed.

Clearly, there are no non-trivial global 1-forms on a numerical Godeaux surface over the complex numbers since $b_{1}=0$. On the other hand, supersingular Godeaux surfaces and Lang's classical Godeaux surfaces in characteristic 5 possess $\alpha_{p^{-}}$, resp. $\mu_{p}$-torsors above them, which implies that they have non-trivial global 1-forms, cf. [CD Proposition I.0.1.11] and Proposition 4.3 below.

\section{PiCARD SCHEME AND Hodge INVARIANTS}

We have seen that non-classical Godeaux surfaces fall into two classes: the singular and the supersingular ones. Also we have seen that these surfaces can exist in characteristic $2 \leq p \leq 5$ only. In this section we specialise to characteristic 5. We will determine their $\mathrm{Pic}^{\tau}$ 's and their Hodge invariants as well as their crystalline cohomology. As we will see, these surfaces behave in many respects like non-classical Enriques surfaces.

Theorem 4.1. Let $X$ be a non-classical Godeaux surface in characteristic 5. Then

$$
\begin{aligned}
& X \text { singular implies } \operatorname{Pic}^{\tau}(X)=\operatorname{Pic}^{0}(X) \cong \mu_{5}, \text { and } \\
& X \text { supersingular implies } \operatorname{Pic}^{\tau}(X)=\operatorname{Pic}^{0}(X) \cong \alpha_{5} .
\end{aligned}
$$

Singular Godeaux surfaces fulfill $\pi_{1}^{\text {ét }}(X) \cong(\mathbb{Z} / 5 \mathbb{Z})$ and supersingular Godeaux surfaces are algebraically simply connected.

Proof. First, we determine $\mathrm{Pic}^{0}$. Assume that $X$ is supersingular. Then we have an inclusion of $\alpha_{5}$ into $G:=\operatorname{Pic}^{0}(X)$ giving rise to an $\alpha_{5}^{D}$-torsor $\pi: Y \rightarrow X$. If $G^{\prime}:=G / \alpha_{5}$ were non-trivial then we would find an embedding of $\alpha_{5}$ or $\mu_{5}$ into $G^{\prime}$, i.e., there would be $\alpha_{5}^{D}$ - or $\mu_{5}^{D}$-torsors above $Y$. In particular, we would have $h^{1}\left(\mathcal{O}_{Y}\right) \neq 0$. We have $K_{Y}^{2}=\chi\left(\mathcal{O}_{Y}\right)=p=5$ by (2), which yields $h^{0}\left(\omega_{Y}\right) \geq 4$. Then Proposition 2.3 forces $h^{0}\left(\omega_{Y}\right) \leq 4$, whence $h^{0}\left(\omega_{Y}\right)=4$ and hence $h^{1}\left(\mathcal{O}_{Y}\right)=0$. This contradiction shows that $G^{\prime}$ is trivial, i.e., $\mathrm{Pic}^{0} \cong \alpha_{5}$. If $X$ is singular, we only have to replace $\alpha_{5}$ by $\mu_{5}$ in the previous discussion.

Next, we show that there is no étale 5 -torsion in $\mathrm{Pic}^{\tau}$. Otherwise, a non-trivial invertible sheaf $\mathcal{L}$ with $\mathcal{L}^{\otimes 5} \cong \mathcal{O}_{X}$ would give rise to a $\mu_{5}$-torsor $\pi: Z \rightarrow X$. As before we conclude $h^{1}\left(\mathcal{O}_{Z}\right)=0$. If $X$ is supersingular, we have an inclusion of $\alpha_{5} \times(\mathbb{Z} / 5 \mathbb{Z})$ into $\mathrm{Pic}^{\tau}$, which yields a non-trivial 
$\alpha_{5}^{D}$-torsor over $Z$. But then $h^{1}\left(\mathcal{O}_{Z}\right) \neq 0$, which we have just excluded. In case $X$ is singular, we argue with $\mu_{5} \times(\mathbb{Z} / 5 \mathbb{Z})$ and conclude as before.

As there is no étale 5-torsion, $Q:=\operatorname{Pic}^{\tau}(X) / \operatorname{Pic}^{0}(X)$ is an étale group scheme of order $\ell(Q)$ prime to 5 . It remains to show that $Q$ is trivial.

If $X$ is singular, then $\mathrm{Pic}^{\tau}$ gives rise to an étale cover of degree $5 \cdot \ell(Q)$. Proposition 1.1 gives $5 \cdot \ell(G) \leq 6$, and hence $Q$ is trivial. Moreover, since we already have an inclusion of $\mathbb{Z} / 5 \mathbb{Z}$ into $\pi_{1}^{\text {ét }}(X)$, Proposition 1.1 tells us that $\pi_{1}^{\text {ét }}(X)$ is in fact isomorphic to $\mathbb{Z} / 5 \mathbb{Z}$.

We will assume from now on that $X$ is supersingular. We know that $\ell(Q) \leq 6$ and $\ell(Q) \neq 5$. If $\ell(Q)=6$ then $Q \cong \mathbb{Z} / 6 \mathbb{Z}$. Hence if we show that there are no elements of order 2 and 3 in $\operatorname{Pic}^{\tau}$, the case $\ell(Q)=6$ is excluded at the same time.

A hypothetical element of order 2 in $\operatorname{Pic}^{\tau}(X)$ would give rise to an étale cover $\varpi: Z \rightarrow X$ of degree 2 and there would still exist a non-trivial $\alpha_{5}$-torsor $Y \rightarrow Z$. We have $\chi\left(\mathcal{O}_{Y}\right)=K_{Y}^{2}=2 \cdot 5=$ 10 and in particular $h^{0}\left(\omega_{Y}\right) \geq 9$. Applying the inequality of Proposition 2.3 to the $\alpha_{5}$-torsor $Y \rightarrow Z$ we obtain a contradiction. Hence there is no non-trivial 2-torsion in $\operatorname{Pic}^{\tau}(X)$. The case of 3-torsion is excluded similarly.

From Proposition 1.1 we know that $\pi_{1}^{\text {ét }}(X)$ is of order at most 6 . Also this group is of order prime to 5 as $X$ is supersingular. If it were non-trivial then it would have to have cyclic quotients of order 2 or 3 . These quotients would give us elements of order 2 or 3 in $\mathrm{Pic}^{\tau}$ which we have just excluded. Hence $\pi_{1}^{\text {ét }}(X)$ is trivial.

Theorem 4.1 together with Proposition 3.4 allows us to compute the Hodge-, the deRham- and the crystalline cohomology groups. The computations for Enriques surfaces presented in [II, Section II.7.3] carry over literally, which is why we omit the proof and only state the result:

Proposition 4.2. Let $X$ be a non-classical Godeaux surface in characteristic 5. Then

$$
h_{\mathrm{dR}}^{1}(X / k)=1, \quad h^{1}\left(\mathcal{O}_{X}\right)=1 \quad \text { and } \quad h^{0}\left(\Omega_{X}^{1}\right)= \begin{cases}0 & \text { if } X \text { is singular } \\ 1 & \text { if } X \text { is supersingular }\end{cases}
$$

The Frölicher spectral sequence degenerates at $E_{1}$-level if and only if the surface is singular. The crystalline cohomology groups are given by

$$
\begin{array}{ccccc}
H_{\text {cris }}^{0}(X / W) & H_{\text {cris }}^{1}(X / W) & H_{\text {cris }}^{2}(X / W) & H_{\text {cris }}^{3}(X / W) & H_{\text {cris }}^{4}(X / W) \\
W & 0 & W^{9} \oplus k & k & W
\end{array}
$$

In any case, the slope spectral sequence degenerates at $E_{1}$-level.

Also, [II, Proposition II.7.3.6] and [II, Proposition II.7.3.8] carry over literally. We only remark that we do not know whether $\rho=b_{2}$ holds true for non-classical Godeaux surfaces but when this result is used in the proof of [II, Proposition II.7.3.6], we only need that $H_{\text {cris }}^{2}(X / W)$ modulo torsion is of slope one, which we have established for numerical Godeaux surfaces in Proposition 3.3

Proposition 4.3. Let $X$ be a non-classical Godeaux surface in characteristic 5 . Then the $E_{1}$-terms of the Frölicher- and the slope spectral spectral sequence are given by 
Frölicher spectral sequence slope spectral sequence

$\begin{array}{lccccccc} & k & 0 & k & k & 0 & W \\ \text { singular } & k & k^{9} & k & 0 & W^{9} & k \\ & k & 0 & k & W & 0 & 0 \\ \text { supersingular } & k & k & k & & & \\ & k & k^{11} & k & k & k & W \\ & k & k & k & & W^{9} & 0 \\ & & & & & & \end{array}$

Finally, we have $H_{\mathrm{dR}}^{2}(X / k)=k^{11}$ and $H_{\mathrm{dR}}^{3}(X / k)=k$.

\section{Classification AND EXAMPles}

In this section we show that non-classical Godeaux surfaces in characteristic 5 are obtained as quotients of quintic hypersurfaces in $\mathbb{P}^{3}$ by a linear fixed point free $\mathbb{Z} / 5 \mathbb{Z}$-action (singular Godeaux surfaces), resp. $\alpha_{5}$-action (supersingular Godeaux surfaces). This is analogous to Reid's classification [Rei, Section 1] of numerical Godeaux surfaces with 5-torsion over the complex numbers. As Miranda [Mir] has already shown existence of singular Godeaux surfaces, we finish this article by establishing the existence of supersingular Godeaux surfaces.

We start with a classification result similar to the one of [La, Section 2] for classical Godeaux surfaces in characteristic 5. The main difficulty is the analysis of supersingular Godeaux surfaces, because the total space of the associated $\alpha_{5}$-torsor is a possibly non-normal surface. If this total space were smooth (which is the case for singular Godeaux surfaces) we could simply argue along the lines of [Hor, Lemma2] and [Hor, Theorem 1].

Theorem 5.1. Let $X$ be a non-classical Godeaux surface in characteristic 5 and $X_{\mathrm{can}}$ its canonical model. We denote by $Y \rightarrow X$ the associated $\mathbb{Z} / 5 \mathbb{Z}$-torsor (in case $X$ is singular), resp. $\alpha_{5}$-torsor (in case $X$ is supersingular). Then there exists a Cartesian diagram

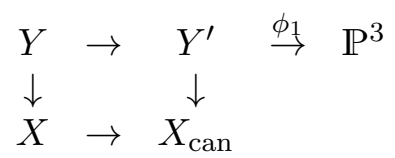

where $Y^{\prime}$ is the canonical model of $Y$. The canonical map of $Y$ is a birational morphism onto a quintic surface in $\mathbb{P}^{3}$. Moreover, it factors over $Y^{\prime}$ and induces an isomorphism of $Y^{\prime}$ with its image. The $\mathbb{Z} / 5 \mathbb{Z}$-, resp. $\alpha_{5}$-action on $Y^{\prime}$ is induced by a linear action on $\mathbb{P}^{3}$.

Remark 5.2. If $X$ is a singular Godeaux surface, then $Y$ is smooth and $Y^{\prime}$, being the canonical model of $Y$, has at worst Du Val singularities. We already noted in Remark 2.5 that $Y$ is not smooth if $X$ is supersingular.

ProOF. Since $X_{\text {can }}$ has only rational singularities we obtain a Frobenius-equivariant isomorphism $H^{1}\left(\mathcal{O}_{X}\right) \cong H^{1}\left(\mathcal{O}_{X_{\text {can }}}\right)$. Hence the associated torsor $Y \rightarrow X$ is the pullback of a torsor of the same type over $X_{\text {can }}$. We denote by $\nu: Y \rightarrow Y^{\prime}$ the morphism induced by this pullback. Being the pullback of an ample invertible sheaf on $X_{\text {can }}$, namely $\omega_{X_{\text {can }}}$ possibly tensorised with an invertible sheaf that is torsion, it follows that $\omega_{Y^{\prime}}$ is ample.

In particular, $Y^{\prime}$ is isomorphic to its canonical model. We get $\omega_{Y} \cong \nu^{*} \omega_{Y^{\prime}}$ and $\nu_{*} \omega_{Y} \cong \omega_{Y^{\prime}}$ by flat base change and the corresponding properties of $\omega_{X}$. Hence $Y^{\prime}$ is the canonical model of $Y$ and the canonical map of $Y$ factors over the canonical map of $Y^{\prime}$. 
We note that here, and in the sequel, we will freely use Ekedahl's results [Ek] about canonical models of surfaces of general type in positive characteristic.

We have $\chi\left(\mathcal{O}_{Y}\right)=5$ and hence $h^{0}\left(\omega_{Y}\right) \geq 4$, whereas Proposition 2.3 tells us $h^{0}\left(\omega_{Y}\right) \leq 4$, whence $h^{0}\left(\omega_{Y}\right)=4$ and $h^{1}\left(\mathcal{O}_{Y}\right)=0$. Let $\varphi_{1}$ be the canonical map of $Y$, which is a possibly rational map to $\mathrm{P}^{3}$, and whose image is a surface by Proposition 2.3

Let $X$ be a supersingular Godeaux surface. We denote by $\rho: X^{(p)} \rightarrow Y$ the map induced by $\pi: Y \rightarrow X$ and the Frobenius morphism. As in the proof of Proposition 2.3, we write the linear subsystem $\rho^{*} H^{0}\left(\omega_{Y}\right)$ of $\left|p K_{X^{(p)}}\right|$ as $|M|+V$. Using that $K_{X}$ is nef, we get $M^{2} \leq\left(p K_{X}\right)^{2}=25$ and using Ekedahl's inequality [Ek, Proposition 0.1.3] we get $M^{2} /\left(p \cdot \operatorname{deg} \varphi_{1}\right) \geq h^{0}\left(\omega_{Y}\right)-2=2$, whence $\operatorname{deg} \varphi_{1} \leq 2$. Suppose we had $\operatorname{deg} \varphi_{1}=2$. Then

$$
25=\left(p K_{X}\right)^{2}=M^{2}+M \cdot V+p K_{X} \cdot V .
$$

First, we show $K_{X} V=0$ (still assuming $\operatorname{deg} \varphi_{1}=2$ ). Suppose that $K_{X} V>0$. Since pluricanonical divisors are connected, we have $M V>0$. Furthermore we have $M^{2} \geq p \operatorname{deg} \varphi_{1} \operatorname{deg}(Z) \geq 20$. Plugging these estimates into (3) we obtain a contradiction. Hence $K_{X} V=0$, which implies that $V$ is a sum of fundamental cycles. Let $C$ be the unique effective divisor in $\left|K_{X}\right|$. Since $F^{*} C$ lies in $\rho^{*} H^{0}\left(\omega_{Y}\right)$, the fixed part $V$ is contained in $F^{*} C$. Let $Z$ be a fundamental cycle in $V$. Then the reduction of $\pi^{*} Z$ is contained in the base locus of $\omega_{Y}$. But $\omega_{Y} \cong \pi^{*} \omega_{X}$, and hence the scheme of base points of $H^{0}\left(\omega_{Y}\right)$ is $\alpha_{p}$-invariant. Hence, $\pi^{*} Z$ is contained in this base scheme from which we conclude that $\rho^{*} \pi^{*} Z=F^{*} Z=p Z$ is contained in $V$. Hence the fixed part is of the form $V=\sum_{i} n_{i} Z_{i}$ for disjoint fundamental cycles $Z_{i}$ and integers $n_{i} \geq p$. We compute $0<M^{2}=$ $\left(p K_{X}-V\right)^{2}=p^{2}-2 \sum_{i} n_{i}^{2}$, which is negative unless $V$ is empty. Hence $V$ is empty, $M^{2}=p^{2}=25$, $\operatorname{deg} \varphi_{1}=2$ and $\operatorname{deg} Z=2$. In particular, $M$ has base points.

Let $\mathfrak{a}:=\operatorname{Bs}\left(\omega_{Y}\right)$ be the ideal sheaf of the base locus of $H^{0}\left(\omega_{Y}\right)$. As $M$ has base points there exists a closed point $y \in Y$ such that $\mathfrak{a} \subseteq \mathfrak{m}_{y}$. We already noted that $\mathfrak{a}$ is $\alpha_{p}$-invariant, from which we conclude that $\mathfrak{a} \subseteq \pi^{-1}\left(\mathfrak{m}_{\pi(y)}\right) \cdot \mathcal{O}_{Y}$. Hence the ideal sheaf of the base locus of $\rho^{*} H^{0}\left(\omega_{Y}\right)$ is contained in $F^{-1}\left(\mathfrak{m}_{\pi(y)}\right) \cdot \mathcal{O}_{X^{(p)}}$. This means that $M$ has a base point of multiplicity $p^{2}$, which is absurd as $M$ defines a morphism onto a surface and $M^{2}=p^{2}$. This contradiction shows that $\varphi_{1}$ cannot be of degree 2 .

Hence $\varphi_{1}$ is birational. Let $C$ be the unique effective divisor of $\left|K_{X}\right|$. Since $K_{X}^{2}=1$, it follows that $C$ is of the form $C^{\prime}+Z$, where $C^{\prime}$ is a reduced and irreducible curve and $Z$ is a sum of fundamental cycles. We write $\rho^{*} H^{0}\left(\omega_{Y}\right)=|M|+V$. As before, the support of $V$ is contained in the support of $C$. If $V$ contains $C^{\prime}$ then $V$ also contains $p C^{\prime}$ (using $\alpha_{p}$-invariance as above) and $M$ is linearly equivalent to a sum of fundamental cycles, which is absurd. Hence $M$ is of the form $p K_{X}-\sum n_{i} Z_{i}$ for disjoint fundamental cycles $Z_{i}$ and integers $n_{i} \geq p$, using again $\alpha_{p}$-invariance. If we assume that the fixed part is not empty, we obtain the contradiction $M^{2}<0$. Hence $V$ is empty and $M^{2}=p^{2}=25$. As base points have to be counted with multiplicity $p^{2}$ and the image of $\varphi_{1}$ is a surface, we conclude that $M$ has no base points. Hence the image $W$ of $\varphi_{1}$ is a quintic hypersurface in $\mathbb{P}^{3}$.

By construction, we can identify $H^{0}\left(\omega_{Y}\right)$ and $H^{0}\left(\mathcal{O}_{W}(1)\right)$. For every integer $n \geq 1$, the map $\mu_{n}$ in the diagram

$$
\begin{array}{ccc}
H^{0}\left(W, \mathcal{O}_{W}(1)\right)^{\otimes n} & \cong & H^{0}\left(Y, \omega_{Y}\right)^{\otimes n} \\
\downarrow \mu_{n} & & \downarrow \mu_{n}^{\prime} \\
H^{0}\left(Z, \mathcal{O}_{W}(n)\right) & -\rightarrow & H^{0}\left(Y, \omega_{Y}^{\otimes n}\right)
\end{array}
$$

is surjective, from which we conclude the existence of the dotted homomorphism making the diagram commutative. Since $\varphi_{1}$ is an isomorphism over an open dense set, we obtain an inclusion of $H^{0}\left(W, \mathcal{O}_{W}(n)\right)$ into $H^{0}\left(Y, \omega_{Y}^{\otimes n}\right)$ for all $n \geq 0$. We have already seen $h^{0}\left(\omega_{Y}\right)=4$. Now, $\omega_{Y}$ is 
the pullback of $\omega_{X}$ possibly tensorised with an invertible sheaf that is torsion. Hence, by the description of $\alpha_{p}$-torsors in [Ek, Proposition I.1.7], we conclude that $\pi_{*}\left(\omega_{Y}^{\otimes n}\right)$ is a successive extension of invertible sheaves on $X$, all of which are numerically equivalent to $\omega_{X}^{\otimes n}$. By Ekedahl's version of Mumford-Ramanujam vanishing [Ek, Proposition II.1.7], the $H^{1}$ of these invertible sheaves on $X$ is zero for all $n \geq 2$. From this we conclude that $h^{1}\left(\omega_{Y}^{\otimes n}\right)=0$ for all $n \geq 2$ and applying RiemannRoch we compute $h^{0}\left(\omega_{Y}^{\otimes n}\right)=5+5 n(n-1) / 2$ for $n \geq 2$. But these are the same dimensions as $h^{0}\left(W, \mathcal{O}_{W}(n)\right)=h^{0}\left(W, \omega_{W}^{\otimes n}\right)$. Hence the injective homomorphism $H^{0}\left(W, \mathcal{O}_{W}(n)\right) \rightarrow H^{0}\left(Y, \omega_{Y}^{\otimes n}\right)$ induced by $\varphi_{1}$ is an isomorphism for all $n \geq 0$. Hence $\varphi_{1}$ induces an isomorphism of the canonical models of $W$ and $Y$, i.e., an isomorphism of $Y^{\prime}$ with $W$, as $W$ is isomorphic to its canonical model.

Since $\mu_{n}$ and $\mu_{n}^{\prime}$ are surjective, it follows that the $\alpha_{p}$-action on $Y^{\prime}$ and $W$ are completely determined by the linear action on $H^{0}\left(Y^{\prime}, \omega_{Y^{\prime}}\right) \cong H^{0}\left(W, \omega_{W}\right)$. In particular, the $\alpha_{p}$-action on $W$ is induced by a linear action on $\mathbb{P}\left(H^{0}\left(W, \omega_{W}\right)^{\vee}\right) \cong \mathbb{P}^{3}$.

If $X$ is a singular Godeaux surface, then $Y$ is a smooth surface. Let $\varpi: \tilde{Y} \rightarrow Y$ be a blow-up such that the movable part $|L|$ of $\left|\varpi^{*} K_{Y}\right|$ has no base points. Since the image of $\varphi_{1}$ is a surface, a generic member $C$ of $L$ is a reduced and irreducible curve, hence $h^{0}\left(C, \mathcal{O}_{C}\right)=1$. We also have $h^{1}\left(\mathcal{O}_{\tilde{Y}}\right)=0$, which then implies $h^{1}\left(\mathcal{O}_{\tilde{Y}}(-C)\right)=0$. Having thus established Kodaira vanishing for $L$ on $\tilde{Y}$ by hand, we can argue as in the proof of [Hor, Lemma 2] to conclude that $\left|K_{Y}\right|$ has no fixed part and at most one base point.

Since $\omega_{Y}$ is the pull-back of an invertible sheaf on $X$, the group $\mathbb{Z} / 5 \mathbb{Z}$ acts on $H^{0}\left(Y, \omega_{Y}\right)$. Hence, if $\left|K_{Y}\right|$ had a base point then the free $(\mathbb{Z} / 5 \mathbb{Z})$-action would give at least 5 base points. Hence $\left|K_{Y}\right|$ has no base points and defines a morphism. Its image $W$ is a surface, and since $K_{Y}^{2}=5$, this map is birational onto a quintic hypersurface in $\mathbb{P}^{3}$. We leave the rest to the reader.

As already mentioned, existence of singular Godeaux surfaces has been settled by Miranda [Mir]:

Theorem 5.3 (Miranda). There do exist singular Godeaux surfaces in characteristic 5.

It remains to establish the existence of supersingular Godeaux surfaces:

Theorem 5.4. There do exist supersingular Godeaux surfaces in characteristic 5.

Proof. On $\mathbb{P}^{3}$ we choose homogeneous coordinates $x_{0}, x_{1}, x_{2}, x_{3}$ and consider the derivation

$$
\delta:=x_{1} \frac{\partial}{\partial x_{0}}+x_{2} \frac{\partial}{\partial x_{1}}+x_{3} \frac{\partial}{\partial x_{2}},
$$

which defines an additive vector field in characteristic 5 , i.e., $\delta^{[5]}=0$. This vector field has precisely one singular point, namely $[1: 0: 0: 0]$. Hence $\delta$ gives rise to an $\alpha_{5}$-action on $\mathbb{P}^{3}$ with one fixed point.

Let $R:=k\left[x_{0}, \ldots, x_{3}\right]$ be the homogeneous coordinate ring of $\mathbb{P}^{3}$ and let $R^{\delta}$ be the fixed ring of $\delta$. Let $V$ be the vector space of elements of degree 5 in $R^{\delta}$. Since $x_{i}^{5} \in V$ for $i=0, \ldots, 3$, the rational map determined by $V$

$$
\varphi: \mathbb{P}^{3} \rightarrow \mathbb{P}(V)
$$

is in fact a morphism. We want to show that $\varphi$ can be identified with the quotient map $\mathbb{P}^{3} \rightarrow \mathbb{P}^{3} / \alpha_{5}$, at least outside the point $[1: 0: 0: 0]$. To do so, we compute in local charts.

We set $x:=x_{0} / x_{3}, y:=x_{1} / x_{3}$ and $z:=x_{2} / x_{3}$ and define $A:=k[x, y, z]$. Then $\delta$ becomes $y \partial_{x}+z \partial_{y}+\partial_{z}$ and we consider

$$
\begin{array}{ll}
\text { homogeneous of degree } 5 & \text { inhomogeneous } \\
F:=x_{1} x_{3}^{4}+2 x_{2}^{2} x_{3}^{3} & f:=y+2 z^{2} \\
G:=-x_{0} x_{3}^{4}+x_{1} x_{2} x_{3}^{3}+3 x_{2}^{3} x_{3}^{2} & g:=-x+y z+3 z^{3},
\end{array}
$$


which lie in the kernel of $\delta$. An easy calculation shows that $B:=A^{5}[f, g]$ is smooth over $k$. The fields of fractions of $A^{\delta}$ and $B$ are purely inseparable extensions of $k\left(x^{5}, y^{5}, z^{5}\right)$ of degree $5^{2}$ and height one. On the other hand, $B$ is contained in $A^{\delta}$ and hence they have the same field of fractions. Moreover, since both rings are normal, they must be equal. Hence, outside $\left\{x_{3}=0\right\}$ the map $\varphi$ induces an isomorphism of its image with $\mathbb{P}^{3} / \alpha_{5}$.

Now, we set $\tilde{x}:=x_{0} / x_{2}, \tilde{y}:=x_{1} / x_{2}$ and $\tilde{z}:=x_{3} / x_{2}$ and define $\tilde{A}:=k[\tilde{x}, \tilde{y}, \tilde{z}]$. Then $\delta$ becomes $(\tilde{y}-\tilde{x} \tilde{z}) \partial_{\tilde{x}}+(1-\tilde{y} \tilde{z}) \partial_{\tilde{y}}-\tilde{z}^{2} \partial_{\tilde{z}}$. Let $\tilde{B}$ be the $\tilde{A}^{5}$-algebra generated by the dehomogenised elements of $V$. We consider $\operatorname{Spec} \tilde{A}^{\delta} \rightarrow \operatorname{Spec} \tilde{B} \rightarrow \operatorname{Spec} \tilde{A}^{5}$. From the computations above we infer that for every closed point of Spec $\tilde{A}^{5}$ whose $\tilde{z}^{5}$-coordinate is not equal to zero, there exists an open neighbourhood over which Spec $\tilde{B}$ and Spec $\tilde{A}^{\delta}$ are isomorphic. Next, we consider the elements

$$
\begin{array}{ll}
\text { homogeneous of degree } 5 & \text { inhomogeneous } \\
x_{3}^{-5} F^{2} & a:=-\tilde{z}-\tilde{y} \tilde{z}^{2}+\tilde{y}^{2} \tilde{z}^{3} \\
x_{3}^{-10}\left(F^{2} G-x_{2}^{5} x_{3}^{5} F\right) & b:=\tilde{x}+2 \tilde{y}^{2}+\tilde{y}^{3} \tilde{z}+\tilde{x} \tilde{y}-\tilde{z} \tilde{y}^{2} \tilde{z}^{2}
\end{array}
$$

which lie in the kernel of $\delta$. We define $\tilde{C}:=\tilde{A}^{5}[a, b]$. Then we have an inclusion $\tilde{C} \subseteq \tilde{B} \subseteq \tilde{A}^{\delta}$ and all three rings have the same field of fractions. A straight forward computation shows that for every closed point of Spec $\tilde{A}^{5}$ with $\tilde{z}^{5}$-coordinate equal to zero, there exists an open neighbourhood, s.th. all closed points of Spec $\tilde{C}$ lying above this neighbourhood are smooth over Spec $k$. Hence over this neighbourhood Spec $\tilde{A}^{\delta}$ and Spec $\tilde{C}$ are isomorphic, and in particular Spec $\tilde{A}^{\delta}$ and Spec $\tilde{B}$ are isomorphic over this neighbourhood. We conclude that $\tilde{B}$ and $\tilde{A}^{\delta}$ are isomorphic and that $\varphi$ can be identified with the quotient map by $\alpha_{5}$ outside $\left\{x_{2}=0\right\}$.

Next, we set $\hat{x}:=x_{0} / x_{1}, \hat{y}:=x_{2} / x_{1}$ and $\hat{z}:=x_{3} / x_{1}$ and define $\hat{A}:=k[\hat{x}, \hat{y}, \hat{z}]$. Then $\delta$ becomes $(1-\hat{x} \hat{y}) \partial_{\hat{x}}+\left(\hat{z}-\hat{y}^{2}\right) \partial_{\hat{y}}-\hat{y} \hat{z} \partial_{\hat{z}}$. We let $\hat{B}$ be the $\hat{A}^{5}$-algebra generated by the dehomogenised elements of $V$. We want to show that Spec $\hat{A}^{\delta}$ and Spec $\hat{B}$ are isomorphic, which - by the previous computations - is clear over the open set of points lying above points of $\operatorname{Spec} \hat{A}^{5}$ with $\hat{y}^{5}$-coordinate or $\hat{z}^{5}$-coordinate not equal to zero. By the previous considerations it is enough to find a $\hat{A}^{5}$-subalgebra $\hat{C}$ of $\hat{B}$ with the same field of fractions such that Spec $\hat{C}$ is smooth over Spec $k$ at all closed points lying above points of Spec $\hat{A}^{5}$ with $\hat{y}^{5}$ - and $\hat{z}^{5}$-coordinate equal to zero. We consider

$$
\begin{array}{ll}
\text { homogeneous of degree } 5 & \text { inhomogeneous } \\
x_{3}^{-15}\left(F^{4}+2 x_{3}^{5} F G^{2}+x_{2}^{5} x_{3}^{10} G\right) & c:=\hat{z}+2 \hat{x}^{2} \hat{z}^{2}+\hat{x} \hat{y} \hat{z}-\hat{x}^{2} \hat{y}^{2} \hat{z} \\
x_{3}^{-15}\left(G^{3} x_{3}^{5}+F^{3} G+F^{2} x_{2}^{5} x_{3}^{5}\right) & d:=\hat{y}-\hat{x} z-\hat{x}^{2} \hat{y}^{3}+3 \hat{x}^{2} \hat{y} \hat{z}-\hat{x}^{3} \hat{z}^{2}+\hat{x} \hat{y}^{2}
\end{array}
$$

which lie in the kernel of $\delta$. An easy computation shows that $\hat{C}:=\hat{A}^{5}[c, d]$ has the desired properties. As before, we conclude that $\varphi$ can be identified with the quotient map by $\alpha_{5}$ outside $\left\{x_{1}=0\right\}$.

Hence $Z:=\operatorname{im}(\varphi)$ coincides with $\mathbb{P}^{3} / \alpha_{5}$ except possibly at $\varphi(P)$, where $P:=[1: 0: 0: 0]$. We have also seen in the computations above that $Z$ is even smooth outside $\varphi(P)$.

Let $X$ be a generic hyperplane section of $Z$, which is a smooth surface since $Z$ has only one isolated singular point. The inverse image $Q$ of $X$ under $\varphi$ in $\mathbb{P}^{3}$ is a $\delta$-invariant quintic and gives rise to an $\alpha_{5}$-torsor $\pi: Q \rightarrow X$.

Being a quintic surface in $\mathbb{P}^{3}$, we compute $\chi\left(\mathcal{O}_{Q}\right)=K_{Q}^{2}=5$, where the self-intersection of the canonical divisor is understood in the sense of formula (1). Using (2) we obtain $\chi\left(\mathcal{O}_{X}\right)=K_{X}^{2}=1$. Since $\omega_{Q}=\pi^{*}\left(\omega_{X}\right)$ is ample, $X$ is a minimal surface of general type, hence a numerical Godeaux surface. The existence of the $\alpha_{5}$-torsor $\pi$ shows that $X$ is supersingular. 


\section{REFERENCES}

[BHPV] W. P. Barth, K. Hulek, C. Peters, A. van de Ven, Compact Complex Surfaces, 2nd edition, Erg. d. Math., 3. Folge Volume 4, Springer (2004).

[Bo] E. Bombieri, Canonical models of surfaces of general type, Inst. Hautes Études Sci. Publ. Math. 42, 171-220 (1973).

[BM2] E. Bombieri, D. Mumford, Enriques' Classification of Surfaces in Char. p, II, Complex analysis and algebraic geometry, Iwanami Shoten, 23-42 (1977).

[BM3] E. Bombieri, D. Mumford, Enriques' Classification of Surfaces in Char. p, III, Invent. math. 35, 197-232 (1976).

[CD] F.R. Cossec, I.V. Dolgachev, Enriques Surfaces I, Prog. in Math. 76, Birkhäuser (1989).

[Cr] R.M. Crew, Étale p-covers in characteristic p, Compositio Math. 52, 31-45 (1984).

[Ek] T. Ekedahl, Canonical models of surfaces of general type in positive characteristic, Inst. Hautes Études Sci. Publ. Math. No. 67, 97-144 (1988).

[Go] L. Godeaux, Sur une surface algébrique de genre zéro et de bigenre deux, Atti Accad. Naz. Lincei, $479-481$ (1931).

[Hor] E. Horikawa, On Deformations of Quintic Surfaces, Invent. math. 31, 43-85 (1975).

[I1] L. Illusie, Complexe de de Rham-Witt et cohomologie cristalline, Ann. Sci. École Norm. Sup. 12, 501-661 (1979).

[La] W.E. Lang, Classical Godeaux Surface in Characteristic p, Math. Ann. 256, 419-427 (1981).

[Lie] C. Liedtke, Algebraic surfaces of general type with small $c_{1}^{2}$ in positive characteristic, arXiv:math/0702548 (2007), to appear in Nagoya Math. J. 191 (2008).

[Mir] R. Miranda, Nonclassical Godeaux surfaces in characteristic five, Proc. Amer. Math. Soc. 91, 9-11 (1984).

[Rei] M. Reid, Surfaces with $p_{g}=0, K^{2}=1$, J. Fac. Sci., Univ. Tokyo, 75-92 (1978).

[Sh] T. Shioda, An Example of Unirational Surfaces in Characteristic p, Math. Ann. 211, 233-236 (1974).

[Sh2] T. Shioda, On Unirationality of Supersingular Surfaces, Math. Ann. 225, 155-159 (1977).

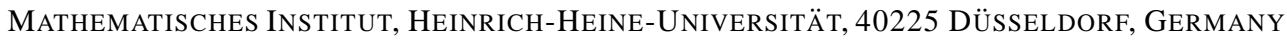

E-mail address: liedtke@math.uni-duesseldorf.de 